\title{
Pacific
}

Journal of

Mathematics

\section{LOCAL COMPARISON THEOREMS FOR KÄHLER} MANIFOLDS

\author{
GANG LIU
}




\title{
LOCAL COMPARISON THEOREMS FOR KÄHLER MANIFOLDS
}

\author{
GANG LIU
}

\begin{abstract}
We establish a sharp relative volume comparison theorem for small balls on Kähler manifolds with lower bound on Ricci curvature, assuming real analyticity of the metric. The model spaces being compared to are complex space forms, that is, Kähler manifolds with constant holomorphic sectional curvature. Moreover, we give an example showing that on Kähler manifolds, the pointwise Laplacian comparison theorem does not hold when the Ricci curvature is bounded from below.
\end{abstract}

\section{Introduction}

Comparison theorems are fundamental tools in geometric analysis. They are vital in estimates of spectra, heat kernels and the Sobolev constants. The classical Bishop-Gromov relative volume comparison theorem [Bishop and Crittenden 1964; Gromov 1981; Li 1993] in Riemannian geometry is this:

Theorem 1.1. Let $M^{n}$ be a complete Riemannian manifold of dimension $n$ such that $\mathrm{Ric} \geq(n-1) K$. For any $p \in M$ and $0<a<b$, the volume of geodesic balls satisfies

$$
\frac{\operatorname{Vol} B_{p}(b)}{\operatorname{Vol} B_{p}(a)} \leq \frac{\operatorname{Vol} B_{M_{K}}(b)}{\operatorname{Vol} B_{M_{K}}(a)},
$$

where $M_{K}$ is the simply connected real space form with sectional curvature $K$ and Vol $B_{M_{K}}(r)$ is the volume of the geodesic ball in $M_{K}$ with radius $r$. Equality holds if and only if $B_{p}(b)$ is isometric to $B_{M_{K}}(b)$.

The key ingredient in Theorem 1.1 is the Laplacian comparison theorem [Cheeger and Ebin 2008; Schoen and Yau 1994]:

Theorem 1.2. Let $M^{n}$ be a complete Riemannian manifold with Ric $\geq(n-1) K$. Let $M_{k}$ be the simply connected real space form with sectional curvature $K$. Denote by $r_{M}(x)$ the distance function from $p$ to $x$ in $M$. Let $r_{M_{k}}$ be the distance function on $M_{k}$. Then for any $x \in M$ and $y \in M_{k}$ with $r_{M}(x)=r_{M_{k}}(y)$,

$$
\Delta r_{M}(x) \leq \Delta r_{M_{k}}(y) \text {. }
$$

MSC2010: primary 53BXX; secondary 53B35, 53B20.

Keywords: volume comparison, Kähler manifolds. 
The model spaces in the theorems above are real space forms. In the Kähler category, a natural question is whether we can replace the model spaces by Kähler models, that is, complex space forms which are Kähler manifolds with constant holomorphic sectional curvature. Li and Wang [2005] showed that when the bisectional curvature has a lower bound, both of the theorems above hold with Kähler models. So the question left is: what can we get if we only assume the lower bound of the Ricci curvature? This note addresses the local case. Our main theorem is:

Theorem 1.3. Let $M^{n}$ be a Kähler manifold of complex dimension $n$ with a real analytic metric. Assume Ric $\geq K$, where $K$ is any real number. Given any point $p \in M$, there exists $r=r(p, M)>0$ such that for any $0<a<b<r$, the volume of geodesic balls satisfies

$$
\frac{\operatorname{Vol} B_{M^{n}}(p, b)}{\operatorname{Vol} B_{M^{n}}(p, a)} \leq \frac{\operatorname{Vol} B_{N_{K}}(b)}{\operatorname{Vol} B_{N_{K}}(a)},
$$

where $N_{K}$ denotes the rescaled complex space form with $\mathrm{Ric}=K$ and $\Delta_{N_{K}} r$ is the Laplacian of distance function on $N_{K}$. Equality holds if and only if $M$ is locally isometric to $N_{K}$.

Remark. Theorem 1.3 is a local version of the Bishop-Gromov relative volume comparison theorem on Kähler manifolds. However, one cannot directly extend the result to any radius. A simple counterexample is a product of several $\mathbb{P}^{1}$ with the standard product metric: the diameter is greater than that of the complex space form. Thus, when $r$ is large, the inequality in Theorem 1.3 does not hold.

We will prove a slightly stronger result:

Theorem 1.4. Under the hypotheses of Theorem 1.3, there exists $r_{0}=r_{0}(p, M)>0$ such that for any $r<r_{0}$, the average Laplacian comparison holds,

$$
\frac{\int_{\partial B_{p}(r)} \Delta r}{A\left(\partial B_{p}(r)\right)} \leq \Delta_{N_{K}} r(r)
$$

where $\Delta_{N_{K}} r$ is the Laplacian of distance function on $N_{K}$. Moreover, the equality holds if and only if $M$ is locally isometric to $N_{K}$.

Remark. Theorem 1.4 is a local version of Theorem 1.2 in the average sense. However, on Kähler manifolds with lower bound on Ricci curvature, the pointwise Laplacian comparison does not even hold locally (see Section 6).

The idea of the proof of Theorem 1.4 is very simple. We shall expand the area of the geodesic sphere $A\left(\partial B_{p}(r)\right)$ as a power series, then compare the coefficients with those of the rescaled complex space form. The computation is complicated since it involves the covariant derivatives of the curvature tensor of arbitrary order.

This note is organized as follows: 
In Section 2, we state two propositions which demonstrate the relation between the derivatives of $A\left(\partial B_{p}(r)\right)$ and the covariant derivatives of the curvature tensor at $p$. Section 3 is the first part of the proof of Proposition 2.1. We shall estimate the derivatives of $A\left(\partial B_{p}(r)\right)$ up to order 4 . In the estimate of the 4-th derivative, the Kähler condition is employed. The most important part is Section 4. We use induction to prove Proposition 2.1. Besides the routine computation, there are two technical lemmas (Lemma 4.4 and Lemma 4.6) which simplify the computation of higher order covariant derivatives of the curvature tensor significantly. One should note that the Kähler condition is essential in these two lemmas. We complete the proof of Proposition 2.2 and Theorem 1.4 in Section 5. The last section is devoted to giving an example showing that the pointwise Laplacian comparison with the complex space form does not necessarily hold if the complex dimension is greater or equal to 2 .

\section{Basic set up}

Throughout this note, we implicitly evaluate derivatives of functions of $r$ at $r=0$. Given a point $p$ on a Kähler manifold $M^{n}$, fix a unit vector $e_{0} \in T_{p} M$. Along the geodesic $l$ from $p$ with initial direction $e_{0}$, consider the Jacobian equation $J^{\prime \prime}=R\left(e_{0}, J\right) e_{0}$. Set up an orthonormal frame $\left\{e_{k}\right\}$ at $p$ such that

$$
J e_{2 i}=e_{2 i+1} \quad \text { and } \quad J e_{2 i+1}=-e_{2 i}
$$

for $0 \leq i \leq n-1$. Parallel transport the frame along the geodesic $l$. Consider the Jacobian field $J_{u}$ with initial value $J_{u}(0)=0, J_{u}^{\prime}(0)=e_{u}$.

We may write

$$
J_{u}=J_{u}\left(r, e_{0}\right)=\sum_{i=1}^{\infty} \sum_{v=0}^{2 n-1} r^{i} C_{u, i}^{v} e_{v}
$$

where $C_{u, i}^{v}$ are constants independent of $r$. Denote $R_{e_{0} e_{u} e_{0} e_{v}}$ by $R_{u v}$ when $e_{0}$ is fixed. Plugging (2-1) into the Jacobian equation, we get

$$
\sum_{i} \sum_{v} i(i-1) r^{i-2} C_{u, i}^{v} e_{v}=\sum_{k} \sum_{w} r^{k} C_{u, k}^{w} R\left(e_{0}, e_{w}\right) e_{0}
$$

Along the geodesic $l$,

$$
R\left(e_{0}, e_{w}\right) e_{0}=\sum_{s=0}^{2 n-1} \sum_{j=0}^{\infty} \frac{R_{s w}^{(j)}}{j !} e_{s} r^{j}
$$

where $R_{s w}^{(j)}$ denotes the $j$-th covariant derivative of $R_{s w}$ along $e_{0}$ at $p$. 
Inserting this into (2-2), we get

$$
\sum_{i, v} i(i-1) r^{i-2} C_{u, i}^{v} e_{v}=\sum_{k, j, w, s} r^{k+j} C_{u, k}^{w} \frac{R_{s w}^{(j)}}{j !} e_{s} .
$$

Comparing coefficients, we obtain

$$
C_{u, i}^{v}=\sum_{k+j=i-2, w} C_{u, k}^{w} \frac{R_{v w}^{(j)}}{j ! i(i-1)} .
$$

A simple iteration now gives the constants $C_{u, i}^{v}$. First we have $C_{u, 1}^{v}=\delta_{u}^{v}$ and $C_{u, 2}^{w}=0$. Then we get

$$
\begin{aligned}
& C_{u, 3}^{v}=\sum_{w} C_{u, 1}^{w} \frac{R_{v w}}{6}=\frac{R_{u v}}{6}, \quad C_{u, 4}^{v}=\sum_{w} C_{u, 1}^{w} \frac{R_{v w}^{\prime}}{12}=\frac{R_{v u}^{\prime}}{12}, \\
& C_{u, 5}^{v}=\sum_{w}\left(C_{u, 1}^{w} \frac{R_{v w}^{\prime \prime}}{40}+C_{u, 3}^{w} \frac{R_{v w}}{20}\right)=\frac{1}{120}\left(\sum_{s} R_{u s} R_{s v}+3 R_{u v}^{\prime \prime}\right) .
\end{aligned}
$$

Plugging these values into (2-1), we have

$$
J_{u}=r e_{u}+\frac{r^{3}}{6} R_{u v} e_{v}+\frac{r^{4}}{12} R_{u v}^{\prime} e_{v}+\frac{r^{5}}{120}\left(\sum_{s} R_{u s} R_{s v}+3 R_{u v}^{\prime \prime}\right) e_{v}+O\left(r^{6}\right) .
$$

We write $d A$ for the standard measure of the unit tangent bundle $U T_{p}(M)$ at $p$, and we write $\int_{\partial B(p, r)} d A$ as $\int$. We define

$$
W=\frac{\int \sqrt{\operatorname{det}\left\langle J_{u}, J_{v}\right\rangle}}{r^{2 n-1}},
$$

and introduce two propositions:

Proposition 2.1. Assume the hypotheses of Theorem 1.3. Let the derivatives of $W$ of order from 1 to $2 m-1$ for $m \geq 1$ be the same as that of the complex space form.

(1) If $m=1,2$, then $\mathrm{Ric}=K$ at $p$.

If $m \geq 3$, then

$$
R_{i \bar{j} k \bar{l}}=\frac{K}{n+1}\left(\delta_{i j} \delta_{k l}+\delta_{i l} \delta_{j k}\right)
$$

at $p$. Moreover, if $u, v, e_{0} \in U T_{p}(M)$ are any unit vectors, then $R_{u v}^{(\lambda)}=0$ for $1 \leq \lambda \leq m-3$ and $\operatorname{Ric}^{(l)}\left(e_{0}, e_{0}\right)=0$ for $1 \leq l \leq 2 m-4$. The superscripts are the orders of covariant derivatives along the direction $e_{0}$.

(2) In either case, $W^{(2 m)}$ is less than or equal to that of the complex space form.

Proposition 2.2. Under the same conditions as in Theorem 1.3, if the derivatives of $W$ of order 1 to $2 m$ for $m \geq 1$ are the same as the complex space form, then $W^{(2 m+1)}=0$.

We divide the proof of Proposition 2.1 into two parts: $m=1,2$ and $m \geq 3$. 


\section{The proof of Proposition 2.1, case $m=1,2$}

By (2-1), we have

$$
\frac{\left\langle J_{u}, J_{v}\right\rangle}{r^{2}}=\sum_{i, j, w} r^{i+j-2} C_{u, i}^{w} C_{v, j}^{w}
$$

By (2-4),

$$
\frac{\left\langle J_{u}, J_{u}\right\rangle}{r^{2}}=1+\frac{1}{3} R_{u u} r^{2}+\frac{1}{6} R_{u u}^{\prime} r^{3}+\left(\frac{2}{45} \sum_{s} R_{u s}^{2}+\frac{1}{20} R_{u u}^{\prime \prime}\right) r^{4}+O\left(r^{5}\right) .
$$

If $u \neq v$,

$$
\frac{\left\langle J_{u}, J_{v}\right\rangle}{r^{2}}=\frac{1}{3} R_{u v} r^{2}+\frac{1}{6} R_{u v}^{\prime} r^{3}+\left(\frac{2}{45} \sum_{s} R_{u s} R_{v s}+\frac{1}{20} R_{u v}^{\prime \prime}\right) r^{4}+O\left(r^{5}\right) .
$$

Now use the above two expressions to see that

$$
\begin{aligned}
\frac{\operatorname{det}\left\langle J_{u}, J_{v}\right\rangle}{r^{4 n-2}} & =1+\frac{1}{3} \sum_{u} R_{u u} r^{2}+\frac{1}{6} \sum_{u} R_{u u}^{\prime} r^{3} \\
& +\left(\frac{2}{45} \sum_{u, s} R_{u s}^{2}+\frac{1}{20} \sum_{u} R_{u u}^{\prime \prime}+\frac{1}{9} \sum_{u<v}\left(R_{u u} R_{v v}-R_{u v}^{2}\right)\right) r^{4}+O\left(r^{5}\right) .
\end{aligned}
$$

Considering the identity $\sqrt{1+x}=1+\frac{1}{2} x-\frac{1}{8} x^{2}+O\left(x^{3}\right)$, we get

$$
\begin{aligned}
& \frac{\sqrt{\operatorname{det}\left\langle J_{u}, J_{v}\right\rangle}}{r^{2 n-1}}=1+\frac{1}{6} \sum_{u} R_{u u} r^{2}+\frac{1}{12} \sum_{u} R_{u u}^{\prime} r^{3} \\
& +\left(\frac{1}{45} \sum_{u, s} R_{u s}^{2}+\frac{1}{40} \sum_{u} R_{u u}^{\prime \prime}+\frac{1}{18} \sum_{u<v}\left(R_{u u} R_{v v}-R_{u v}^{2}\right)-\frac{1}{72}\left(\sum_{u} R_{u u}\right)^{2}\right) r^{4}+O\left(r^{5}\right) .
\end{aligned}
$$

Since $W=\frac{1}{r^{2 n-1}} \int \sqrt{\operatorname{det}\left\langle J_{u}, J_{v}\right\rangle}$, we find

$$
W^{\prime}(0)=0 \quad \text { and } \quad W^{\prime \prime}(0)=-c s,
$$

where $c$ is a positive constant depending only on $n$, and $s$ is the scalar curvature at $p$. Therefore $W^{\prime \prime}(0)$ is less than or equal to that of the complex space form. This proves Proposition 2.1 for $m=1$.

Now we consider $m=2$. According to the assumption of Proposition 2.1, $W^{\prime \prime}$ is the same as that of the complex space form. Therefore $s=n K$ at $p$. Since the Ricci curvature is bounded from below by $K$, Ric $=K g$ at $p$. By (3-3), it is simple to see that the $r^{3}$ coefficient of $W$ is 0 by symmetry. Thus to complete the proof for $m=2$, we just need to show that the 4th derivative of $W$ is less than or equal to that of the complex space form. 
We keep in mind that Ric $=K g$ at $p$. The $r^{4}$ coefficient of $W$ is

$$
\begin{aligned}
c_{4}= & \int\left(\frac{1}{45} \sum_{u, s} R_{u s}^{2}+\frac{1}{40} \sum_{u} R_{u u}^{\prime \prime}+\frac{1}{18} \sum_{u<v}\left(R_{u u} R_{v v}-R_{u v}^{2}\right)-\frac{1}{72}\left(\sum_{u} R_{u u}\right)^{2}\right) \\
= & \frac{1}{360} \int\left(8 \sum_{u} R_{u u}^{2}+16 \sum_{u<v} R_{u v}^{2}+9 \sum_{u} R_{u u}^{\prime \prime}\right. \\
& \left.+20 \sum_{u<v} R_{u u} R_{v v}-20 \sum_{u<v} R_{u v}^{2}-5\left(\sum_{u} R_{u u}\right)^{2}\right) \\
= & \frac{1}{360} \int\left(-2 \sum_{u} R_{u u}^{2}+10\left(\sum_{u} R_{u u}\right)^{2}-4 \sum_{u<v} R_{u v}^{2}+9 \sum_{u} R_{u u}^{\prime \prime}-5\left(\sum_{u} R_{u u}\right)^{2}\right) \\
= & \frac{1}{360} \int\left(9 \sum_{u} R_{u u}^{\prime \prime}-4 \sum_{u<v} R_{u v}^{2}-2 \sum_{u} R_{u u}^{2}+5\left(\sum_{u} R_{u u}\right)^{2}\right) .
\end{aligned}
$$

Note that the Ricci curvature attains the minimum $K$ at $p$, so

$$
\sum_{u} R_{u u}^{\prime \prime}=-\operatorname{Ric}^{\prime \prime}\left(e_{0}, e_{0}\right) \leq 0
$$

Therefore we have

$$
\begin{aligned}
c_{4} & =\frac{1}{360} \int\left(9 \sum_{u} R_{u u}^{\prime \prime}-4 \sum_{u<v} R_{u v}^{2}-2 \sum_{u} R_{u u}^{2}+5 K^{2}\right) \\
& \leq-\frac{1}{360} \int\left(2 \sum_{u} R_{u u}^{2}-5 K^{2}\right) \\
& =-\frac{1}{360} \int\left(2 \sum_{u \neq 1} R_{u u}^{2}+2 R_{11}^{2}-5 K^{2}\right) \\
& \leq-\frac{1}{360} \int\left(\frac{1}{n-1}\left(\sum_{u \neq 1} R_{u u}\right)^{2}+2 R_{11}^{2}-5 K^{2}\right) \\
& =-\frac{1}{360} \int\left(\frac{1}{n-1}\left(\operatorname{Ric}\left(e_{0}, e_{0}\right)+R_{11}\right)^{2}+2 R_{11}^{2}-5 K^{2}\right) \\
& =-\frac{1}{360} \int\left(\frac{1}{n-1} K^{2}+\frac{2}{n-1} K R_{11}+\left(\frac{1}{n-1}+2\right) R_{11}^{2}-5 K^{2}\right) \\
& \leq-\frac{1}{360}\left(\int \frac{1}{n-1} K^{2}+\frac{2}{n-1} K \int R_{11}+C_{1}\left(\int R_{11}\right)^{2}-\int 5 K^{2}\right) \\
& =C_{2} K^{2},
\end{aligned}
$$

where $C_{1}, C_{2}$ are constants depending only on $n$.

We explain the inequalities above. In the first inequality, we drop the two terms $\sum_{u<v} R_{u v}^{2}$ and $\sum_{u} R_{u u}^{\prime \prime}$. In the second inequality, we apply the Schwartz inequality for directions $e_{u}$ that are perpendicular to $e_{1}, e_{0}$. In the third inequality we use the Schwartz inequality $\int R_{11}^{2} \geq C\left(\int R_{11}\right)^{2}$. We make use of the Kähler condition to 
obtain $\int R_{11}=C_{3} s=n C_{3} K$, where $C_{3}$ is a constant depending only on $n$. This explains the last equality.

The right hand side of (3-4) is exactly the case of the complex space form. Therefore when $W^{\prime}$ and $W^{\prime \prime}$ are the same as the complex space form, $W^{(3)}=0$ and $W^{(4)}$ is less than or equal to that of the complex space form. Equation (3-4) becomes an equality if and only if the holomorphic sectional curvature is constant at $p$ and $\operatorname{Ric}^{\prime \prime}\left(e_{0}, e_{0}\right)=0$ for any $e_{0} \in U T_{p} M$. This completes the proof for $m=2$.

\section{The proof of Proposition 2.1, case $m \geq 3$}

Denote $\operatorname{Ric}^{(l)}\left(e_{0}, e_{0}\right)$ by $\operatorname{Ric}^{(l)}$. According to the assumption of Proposition 2.1, the derivatives of $W$ of order 1 to $2 m-1$ are the same as the complex space form. From results in the last section, the holomorphic sectional curvature is constant at $p$ and $\mathrm{Ric}^{\prime \prime}=0$ for any $e_{0}$. That is to say,

$$
R_{i \bar{j} k \bar{l}}=\frac{K}{n+1}\left(\delta_{i j} \delta_{k l}+\delta_{i l} \delta_{j k}\right) \quad \text { and } \quad \operatorname{Ric}^{\prime \prime}=0
$$

at $p$. Therefore, we have proved part (1) of Proposition 2.1 for $m=3$.

Now we use induction. Assuming that part (1) of Proposition 2.1 holds for $k=m$, we shall prove that it holds for $k=m+1$.

Claim 4.1. Let $C_{u, i}^{v}$ be the coefficients defined in (2-1) for $i \leq m$. Under the hypothesis of the induction above, $C_{u, i}^{v}$ are constants independent of the direction $e_{0}$. In fact, they are the same as that of the complex space form.

Proof. The claim follows if we insert the induction hypothesis into (2-3).

Let us write

$$
\frac{\operatorname{det}\left\langle J_{u}, J_{v}\right\rangle}{r^{4 n-2}}=1+\sum_{i=1}^{m-1} a_{i} r^{i}+\sum_{j=m}^{2 m} b_{j} r^{j}+O\left(r^{2 m+1}\right) .
$$

Combining Claim 4.1 with (3-1), we find that $a_{i}$ are constants independent of the direction $e_{0}$. Equation (3-1) also yields $C_{u, m+1}^{v}=C_{v, m+1}^{u}$ for all $u, v$. Direct expansion of the determinant via (3-1) gives

$$
\begin{aligned}
b_{2 m}=\sum_{u, v}\left(C_{u, m+1}^{v}\right)^{2}+ & 4 \sum_{u<v} C_{u, m+1}^{u} C_{v, m+1}^{v}+2 \sum_{u} C_{u, 2 m+1}^{u} \\
& -4 \sum_{u<v} C_{u, m+1}^{v} C_{v, m+1}^{u}+\sum_{i=1}^{m} C_{u, m+i}^{v} C_{i, m, u, v}+C_{0, m}
\end{aligned}
$$

where $C_{i, m, u, v}$ and $C_{0, m}$ are all constants independent of the direction $e_{0}$.

Note also

$$
b_{m}=2 \sum_{u} C_{u, m+1}^{u}+\text { Constant } .
$$


Let us set $\gamma_{m}=\sum_{i=1}^{m-1} a_{i} r^{i}+\sum_{j=m}^{2 m} b_{j} r^{j}$ for $m \geq 1$. Applying the Taylor series expansion

$$
\sqrt{1+x}=1+\frac{1}{2} x-\frac{1}{8} x^{2}+\sum_{k=3}^{\infty} \lambda_{k} x^{k}
$$

for $|x|<1$, we obtain

$$
\frac{\sqrt{\operatorname{det}\left\langle J_{u}, J_{v}\right\rangle}}{r^{2 n-1}}=1+\frac{1}{2} \gamma_{m}-\frac{1}{8} \gamma_{m}^{2}+\sum_{k=3}^{\infty} \lambda_{k} \gamma_{m}^{k}+O\left(r^{2 m+1}\right) .
$$

Lemma 4.2. The $2 m$-th order coefficient of the expansion of $W$ is

$$
\begin{aligned}
c_{2 m}= & \int\left(\frac{1}{2} \sum_{u, v}\left(C_{u, m+1}^{v}\right)^{2}+2 \sum_{u<v} C_{u, m+1}^{u} C_{v, m+1}^{v}+\sum_{u} C_{u, 2 m+1}^{u}\right. \\
& \left.-2 \sum_{u<v} C_{u, m+1}^{v} C_{v, m+1}^{u}-\frac{1}{2}\left(\sum_{u} C_{u, m+1}^{u}\right)^{2}+\sum_{i=1}^{m} C_{u, m+i}^{v} \widetilde{C}_{i, m, u, v}\right)+\widetilde{C}_{0, m}
\end{aligned}
$$

where $\widetilde{C}_{i, m, u, v}$ and $\widetilde{C}_{0, m}$ are constants independent of the direction $e_{0}$.

Proof. It suffices to find out the contribution of each term in (4-4) to $c_{2 m}$. We keep in mind that coefficients $a_{i}$ in (4-1) are independent of $e_{0}$.

By (4-2), the contribution of the term $1+\frac{1}{2} \gamma_{m}$ to $c_{2 m}$ is

$$
\begin{aligned}
\int \frac{1}{2} \sum_{u, v}\left(C_{u, m+1}^{v}\right)^{2}+ & 2 \sum_{u<v} C_{u, m+1}^{u} C_{v, m+1}^{v}+\sum_{u} C_{u, 2 m+1}^{u} \\
& -2 \sum_{u<v} C_{u, m+1}^{v} C_{v, m+1}^{u}+\frac{1}{2}\left(\sum_{i=1}^{m} C_{u, m+i}^{v} C_{i, m, u, v}+C_{0, m}\right) .
\end{aligned}
$$

The contribution of the term $-\frac{1}{8} \gamma_{m}^{2}$ to $c_{2 m}$ is

$$
-\int\left(\frac{1}{8} b_{m}^{2}+\sum_{i=1}^{m} C_{u, m+i}^{v} p_{i, m, u, v}\right)+p_{0, m} .
$$

By (4-3), it could be written as

$$
-\int\left(\frac{1}{2}\left(\sum_{u} C_{u, m+1}^{u}\right)^{2}+\sum_{i=1}^{m} C_{u, m+i}^{v} p_{i, m, u, v}\right)+p_{0, m} .
$$

The contribution of $\sum_{k=3}^{\infty} \lambda_{k} \gamma_{m}^{k}$ to $c_{2 m}$ is

$$
\int \sum_{i=1}^{m} C_{u, m+i}^{v} q_{i, m, u, v}+q_{0, m} .
$$

In (4-7), (4-8), (4-9), $p_{i, m, u, v}, q_{i, m, u, v}, p_{0, m}$ and $q_{0, m}$ are all constants independent of the direction $e_{0}$. Lemma 4.2 follows if we combine (4-6), (4-7), (4-8) and (4-9). 
Lemma 4.3. There is a negative definite quadratic form $Q$, constants $h_{m, i}$ and $C$ and a negative constant $C_{m}$ such that

$$
c_{2 m}=\int Q\left(R_{u v}^{(m-2)}\right)+\sum_{i=-2}^{m-4} h_{m, i} \int R_{11}^{(m+i)}+C_{m} \int \operatorname{Ric}^{(2 m-2)}+C .
$$

Proof. By the induction hypothesis and (2-3), we have

$$
\begin{aligned}
& C_{u, 2 m+1}^{u} \\
= & \sum_{k+j=2 m-1, w} \frac{C_{u, k}^{w} R_{u w}^{(j)}}{j !(2 m+1) 2 m} \\
= & \frac{1}{(2 m+1) 2 m}\left(\sum_{w}\left(\frac{R_{u w}^{(m-2)} C_{u, m+1}^{w}}{(m-2) !}+\sum_{j=m-1}^{2 m-2} B_{j, m, w, u} R_{u w}^{(j)}\right)+R_{u u} C_{u, 2 m-1}^{u}\right)
\end{aligned}
$$

where $B_{j, m, w, u}$ are constants. For $i \leq m$, we have

$$
C_{u, m+i}^{v}=\sum_{j=m-2}^{m+i-3} d_{m, i, j, w, u} R_{u w}^{(j)}+C
$$

where $C$ and $d_{m, i, j, w, u}$ are constants. In particular, we have

$$
C_{u, m+1}^{v}=\sum_{k+j=m-1, w} C_{u, k}^{w} \frac{R_{v w}^{(j)}}{j ! m(m+1)}=\frac{1}{m(m+1)}\left(\frac{R_{v u}^{(m-2)}}{(m-2) !}+C_{u, m-1}^{v} R_{v v}\right)
$$

By the induction hypothesis,

$$
\sum_{u} R_{u u}^{(m-2)}=-\operatorname{Ric}^{(m-2)}=0 .
$$

Therefore

$$
\begin{aligned}
\sum_{u}\left(R_{u u}^{(m-2)}\right)^{2} & =\left(\sum_{u} R_{u u}^{(m-2)}\right)^{2}-2 \sum_{u<v} R_{u u}^{(m-2)} R_{v v}^{(m-2)} \\
& =-2 \sum_{u<v} R_{u u}^{(m-2)} R_{v v}^{(m-2)} .
\end{aligned}
$$

Inserting (4-11), (4-12), (4-13) into (4-5), we find

$$
c_{2 m}=\int Q\left(R_{u v}^{(m-2)}\right)+\sum_{i=-2}^{m-2} \int \sum_{u, v} h_{m, i, u, v} R_{u v}^{(m+i)}+C .
$$

Now we prove that $Q$ is negative definite. Let us check each term in (4-5). By (4-13), the term $\frac{1}{2} \sum_{u, v}\left(C_{u, m+1}^{v}\right)^{2}$ in (4-5) contributes to the quadratic term

$$
\sum_{u, v} \frac{1}{2 m^{2}(m+1)^{2}((m-2) !)^{2}}\left(R_{u v}^{(m-2)}\right)^{2} .
$$


The term $2 \sum_{u<v} C_{u, m+1}^{u} C_{v, m+1}^{v}$ contributes to the quadratic term

$$
\sum_{u<v} \frac{2}{m^{2}(m+1)^{2}((m-2) !)^{2}} R_{u u}^{(m-2)} R_{v v}^{(m-2)} .
$$

By (4-15), it could be written as

$$
-\frac{1}{m^{2}(m+1)^{2}((m-2) !)^{2}} \sum_{u}\left(R_{u u}^{(m-2)}\right)^{2} .
$$

By (4-11) and (4-13), the term $\sum_{u} C_{u, 2 m+1}^{u}$ contributes to the quadratic term

$$
\sum_{u, v} \frac{1}{2 m^{2}(m+1)(2 m+1)((m-2) !)^{2}}\left(R_{u v}^{(m-2)}\right)^{2} .
$$

The term $-2 \sum_{u<v} C_{u, m+1}^{v} C_{v, m+1}^{u}$ contributes to the quadratic term

$$
-\sum_{u<v} \frac{2}{m^{2}(m+1)^{2}((m-2) !)^{2}}\left(R_{u v}^{(m-2)}\right)^{2} .
$$

The term $-\frac{1}{2}\left(\sum_{u} C_{u, m+1}^{u}\right)^{2}$ is obviously negative semidefinite.

By combining (4-17), (4-18), (4-19), (4-20) and (4-21), it follows that the quadratic form in (4-10) is negative definite.

Consider the linear terms in (4-16). By the induction hypothesis, the coefficients $h_{m, i, u, v}$ are unchanged if we take a unitary transformation keeping the direction $e_{0}$ fixed. Comparing the coefficients of the linear order terms, we see that $h_{m, i, u, v}=0$ if $u \neq v$, and $h_{m, i, u, u}=h_{m, i, v, v}$ if $u \neq e_{1}$ and $v \neq e_{1}$. Therefore, the linear terms $h_{m, i, u, u} R_{u u}^{(m+i)}$ could be absorbed into Ric ${ }^{(m+i)}$ with the terms $-h_{m, i} R_{11}^{(m+i)}$ left. Also note that by induction hypothesis, $\operatorname{Ric}^{(l)}=0$ for $0<l \leq 2 m-3$ (the term $\operatorname{Ric}^{(2 m-3)}$ vanishes as the Ricci curvature attains its minimum at $p$ ). Finally, one verifies that $\sum_{u} C_{u, 2 m+1}^{u}$ is the only term in (4-5) that has contribution to $R_{u v}^{(2 m-2)}$. Therefore the linear terms in (4-16) could be written as

$$
\sum_{i=-2}^{m-4} h_{m, i} \int R_{11}^{(m+i)}+C_{m} \int \operatorname{Ric}^{(2 m-2)}
$$

From (4-11), it is simple to check that $C_{m}$ is negative.

By the induction hypothesis and that the Ricci curvature attains its minimum at $p$, we have $\operatorname{Ric}^{(2 m-2)} \geq 0$. It follows from Lemma 4.3 that

$$
c_{2 m} \leq \sum_{i=-2}^{m-4} h_{m, i} \int R_{11}^{(m+i)}+\text { Constant } .
$$

We would like to prove that the linear terms $\int R_{11}^{(m+i)}$ vanish for $-2 \leq i \leq m-4$. Note that by symmetry, if $m+i$ is odd, the integral equals 0 . Let us deal with case 
when $m+i$ is even. We shall check when $i=m-4$. The other cases are similar. Let

$$
A=-\frac{1}{4} \int R_{11}^{(2 m-4)}
$$

Set up an orthonormal frame $\left\{f_{i}\right\}$ at $p$ such that $J f_{2 j}=f_{2 j+1}$ and $J f_{2 j+1}=-f_{2 j}$ for $0 \leq j \leq n-1$. Letting $\beta_{j}=\frac{1}{2}\left(f_{2 j}-\sqrt{-1} f_{2 j+1}\right)$, in a small neighborhood of $p$, we parallel transport the frame along each geodesic through $p$. Suppose that

$$
e_{0}=\sum_{j=0}^{n-1}\left(z_{j} \beta_{j}+\overline{z_{j} \beta_{j}}\right) \text {. }
$$

Lemma 4.4. Under the assumption of the induction in Proposition $2.1, R m^{(\lambda)}=0$ at $p$ for $1 \leq \lambda \leq m-3$, where $R m^{(\lambda)}$ denotes any covariant derivative of the curvature tensor of order $\lambda$ at $p$.

Proof. We use induction. If $\lambda=0$, the result automatically holds since there is nothing to prove. Suppose the result holds for $k<\lambda$. For $k=\lambda$, we plug (4-24) in $R_{u v}^{(\lambda)}$.

Claim 4.5. We can commute the covariant derivatives of $R_{u v}^{(\lambda)}$.

Proof. To prove the claim, we only need to consider the case $\lambda \geq 2$. By the induction hypothesis of Lemma 4.4, the covariant derivatives of the curvature tensor vanish up to order $\lambda-1$ at $p$. If $\lambda>3$, the claim follows from the Ricci identity. Now suppose $\lambda=2$. By the Ricci identity, the difference of commuting the covariant derivatives is a function of the curvature tensor. Note that the curvature tensor at $p$ is the same as of the complex space form. This completes the proof for $\lambda=2$.

We insert (4-24) into $R_{J e_{0} J e_{0}}^{(\lambda)}$. By Claim 4.5 and the Bianchi identities, $R_{J e_{0} J e_{0}}^{(\lambda)}$ becomes a polynomial in the variables $z_{j}, \bar{z}_{j}$. The coefficients of the polynomial are exactly all the covariant derivatives of $R m$ at $p$ of order $\lambda$. According to the assumption of Lemma 4.4, $R_{J e_{0} J e_{0}}^{(\lambda)}$ is identically 0 for all $e_{0}$. Therefore, the coefficients of the polynomial are all 0 . This completes the induction of Lemma 4.4.

Lemma 4.6. Under the assumption of the induction in Proposition 2.1, A could be written as $\sum_{i=1}^{m-2} g_{i, m} \Delta^{i} s$, where $s$ denotes the scalar curvature, and $g_{i, m}$ are constants depending only on $n, m$ and $i$.

Proof. Define $X=\frac{1}{2}\left(e_{0}-\sqrt{-1} J e_{0}\right)$, then $A=\int R_{X \bar{X}} X \bar{X}, e_{0} e_{0} \ldots e_{0}$, where the number of $e_{0}$ is $2 m-4$. Integrating and plugging (4-24) into the result, we find

$$
A=\sum_{\alpha_{1} \alpha_{2} \ldots \alpha_{2 m}}\left(\int \alpha_{1} \alpha_{2} \ldots \alpha_{2 m}\right) R_{\alpha_{1} \alpha_{2} \ldots \alpha_{2 m}}
$$


where each $\alpha_{i}$ is either $z_{j}$ or $\bar{z}_{k}$ for $0 \leq j, k \leq n-1$, with the further condition that $\alpha_{1}, \alpha_{3} \in\left\{z_{j}\right\}$, and $\alpha_{2}, \alpha_{4} \in\left\{\overline{z_{k}}\right\}$. Under the subscript of $R, z_{j}$ stands for $\beta_{j}$, and $\bar{z}_{k}$ stands for $\bar{\beta}_{k}$.

From the expression of (4-25), we see that $z_{i}, \bar{z}_{i}$ must all go in pairs in the sequence $\alpha_{1} \alpha_{2} \ldots \alpha_{2 m}$, otherwise the integral $\int \alpha_{1} \alpha_{2} \ldots \alpha_{2 m}$ would equal 0 . Using the Kähler identities, we can switch the covariant derivatives in (4-25) and rearrange it as

$$
A=\sum_{I_{1}, I_{2}, \ldots I_{n}} C_{I_{1} I_{2} \ldots I_{n}} R_{I_{1} I_{2} \ldots I_{n}}+B
$$

Here the symbol $I_{j}$ denotes $z_{j} \bar{z}_{j} \ldots z_{j} \bar{z}_{j}$; we have $\sum_{j}\left|I_{j}\right|=2 m$; subscripts after the fourth subscript of $R$ denote covariant derivatives; $C_{I_{1} I_{2} \ldots I_{n}}$ are the coefficients in (4-25); and $B$ is a combination of covariant derivatives of $R m$ of lower order.

From (4-23), we see that the coefficients $C_{I_{1} I_{2} \ldots I_{n}}$ in (4-26) are unitary invariants. For fixed $I_{3}, I_{4}, \ldots, I_{n}$, let $d=\left|I_{1}\right|+\left|I_{2}\right|$. Denote the coefficient $C_{I_{1} I_{2} \ldots I_{n}}$ by $C_{p}$, where $0 \leq\left|I_{1}\right|=p \leq d$. We want to find relations between the different $C_{p}$. Define a unitary transformation by setting $\widetilde{\beta_{i}}=\beta_{i}$ for $i \neq 1,2$ and let

$$
\beta_{1}=\cos \theta \widetilde{\beta_{1}}+\sin \theta \widetilde{\beta_{2}} \quad \text { and } \quad \beta_{2}=-\sin \theta \widetilde{\beta_{1}}+\cos \theta \widetilde{\beta_{2}} .
$$

Insert the unitary transformation above in (4-26). Then the new coefficient $\tilde{C}_{d}$ becomes $\sum_{p=0}^{d} C_{p} \cos ^{2 p} \theta \sin ^{2(d-p)} \theta$. Therefore we have:

$$
\sum_{p=0}^{d} C_{p} \cos ^{2 p} \theta \sin ^{2(d-p)} \theta=C_{d}=C_{d}\left(\cos ^{2} \theta+\sin ^{2} \theta\right)^{d} .
$$

Claim 4.7. $C_{p}=C_{d}\left(\begin{array}{l}d \\ p\end{array}\right)$.

Proof. Divide by $\cos ^{2 d} \theta$ on both sides, then (4-27) becomes

$$
\sum_{p=0}^{d} C_{p} \tan ^{2(d-p)} \theta=C_{d}=C_{d}\left(1+\tan ^{2} \theta\right)^{d} .
$$

Since $\theta$ is arbitrary, the claim follows.

By Claim 4.7, $C_{p} / C_{d}=\left(\begin{array}{l}d \\ p\end{array}\right)$. Since we can substitute any index $u, v$ for 1,2 , the ratios of all coefficients in (4-26) are determined. Note that to get the relations between $C_{p}$, we only use the condition that the form (4-23) is unitary invariant. Since $\Delta^{m-2} s$ is also unitary invariant with respect to the frame, we can write it in the same form as (4-26). By the same argument, the ratios of coefficients of $\Delta^{m-2} s$ are the same as of coefficients in (4-26). It follows that the term $\sum_{I_{1}, I_{2}, \ldots I_{n}} C_{I_{1} I_{2} \ldots I_{n}} R_{I_{1} I_{2} \ldots I_{n}}$ in (4-26) equals $C(m, n) \Delta^{(m-2)} s$ modulo lower order covariant derivatives, where $C(m, n)$ is a constant depending only on $m, n$. 
Now we make an important observation. From the Ricci identity,

$$
R_{i_{1} \bar{i}_{2} \ldots i_{p} \alpha \beta i_{p+3} \ldots i_{2 m}}-R_{i_{1} \bar{i}_{2} \ldots i_{p} \beta \alpha i_{p+3} \ldots i_{2 m}}
$$

is the sum of $\left(R m R m^{(p-4)}\right)_{, i_{p+3} \ldots i_{2 m}}$. By Lemma $4.4, R m^{(\lambda)}=0$ for $1 \leq \lambda \leq m-3$. It follows that $\left(R m R m, i_{5} \ldots i p\right), i_{p+3} \ldots i_{2 m}$ can be expanded as a linear combination of the covariant derivatives of curvature tensor. Therefore $A-C(m, n) \Delta^{(m-2)} s$ can be written as a linear combination of the covariant derivatives of the curvature tensor with the highest order $2 m-6$. Furthermore it is unitary invariant since the curvature tensor is unitary invariant at $p$. By induction, we have completed the proof of Lemma 4.6.

From the induction in Proposition 2.1, $\operatorname{Ric}^{(l)}=0$ for $1 \leq l \leq 2 m-4$. Integrating over the unit sphere in $T_{p} M$ we find, by similar arguments as in the proof of Lemma 4.6, that for $l$ even

$$
0=\int \operatorname{Ric}_{e_{0} e_{0}, e_{0} e_{0} \ldots e_{0}}=\sum_{k=1}^{l / 2} C_{l, k} \Delta^{k} s
$$

where the order of the covariant derivative above is $l$. It is straightforward to check that the highest order coefficient $C_{l, l / 2}$ is not equal to 0 . Then, by induction, $\Delta^{k} s=0$ at $p$ for $1 \leq k \leq m-2$. Combining this with Lemma 4.6, it follows that $A=0$. Similarly all linear terms in (4-10) vanish. Therefore, under the induction hypothesis in Proposition 2.1, in order that $c_{2 m}$ in (4-10) achieves the maximum, we must have $\operatorname{Ric}^{(2 m-2)}=0$ and $R_{u v}^{(\lambda)}=0$ for $1 \leq \lambda \leq m-2$. This is exactly the case of the complex space form. Therefore we have completed the induction step for part (1) in Proposition 2.1 and, as a byproduct, we have proved part (2) as well. The proof is thus complete.

\section{The proof of Theorem 1.4}

Proof of Proposition 2.2. Using the same argument as in the last section, we find that $W^{(2 m+1)}$ is a linear combination of $\int R_{11}^{(m+i)}$ for $1 \leq i \leq m-3$ (the terms of order greater than $2 m-3$ can be absorbed into $\left.\operatorname{Ric}^{(m+i)}\right)$. Then $W^{(2 m+1)}$ is equal to 0 by similar arguments as in the proof of Lemma 4.6.

Proof of Theorem 1.4. Consider the two cases below:

1. All coefficients of the power series of $W$ are equal to that of the complex space form. From Proposition 2.1, all covariant derivatives of the curvature tensor at $p$ are the same as the complex space form. Since the metric is real analytic, we conclude that near $p$, the manifold is isometric to the complex space form.

2. There is a $i_{0} \geq 1$ such that for all $i<i_{0}$, the coefficients of the power series of $W$ are equal to that of the complex space form, but the $i_{0}$-th coefficient is less than that of the complex space form. Checking the power series of $W^{\prime} / W$ at $p$, 
we find that for sufficiently small $r, W^{\prime} / W$ is less than that of the complex space form. From the definition of $W$ we have, for small $r$,

$$
\frac{\int_{\partial B_{p}(r)} \Delta r}{A\left(\partial B_{p}(r)\right)}=\frac{\int \sqrt{\operatorname{det}\left\langle J_{u}, J_{v}\right\rangle}}{\int \sqrt{\operatorname{det}\left\langle J_{u}, J_{v}\right\rangle}}<\Delta_{N_{K}} r(r) .
$$

\section{An example}

In this section we give an example showing that the analogous Laplacian comparison theorem is not true on Kähler manifolds when the Ricci curvature is bounded from below by a nonzero constant. The example is in dimension 2. For higher dimensions, the construction is similar.

Identify $\mathbb{R}^{4}$ with $\mathbb{C}^{2}$ in the usual way. The corresponding almost complex structure $J$ is given by

$$
J \frac{\partial}{\partial x_{1}}=\frac{\partial}{\partial x_{2}}, \quad J \frac{\partial}{\partial x_{2}}=-\frac{\partial}{\partial x_{1}}, \quad J \frac{\partial}{\partial x_{3}}=\frac{\partial}{\partial x_{4}} \quad \text { and } \quad J \frac{\partial}{\partial x_{4}}=-\frac{\partial}{\partial x_{3}} .
$$

Given a small ball near the origin of $\mathbb{C}^{2}$, define a function $f$ to be

$$
\begin{aligned}
f=\left|z_{1}\right|^{2}+\left|z_{2}\right|^{2}+a\left|z_{1}\right|^{4}+8 a\left|z_{1}\right|^{2}\left|z_{2}\right|^{2}+a\left|z_{2}\right|^{4}+\frac{8}{3} a^{2}\left|z_{1}\right|^{6} \\
+28 a^{2}\left|z_{1}\right|^{4}\left|z_{2}\right|^{2}+28 a^{2}\left|z_{1}\right|^{2}\left|z_{2}\right|^{4}+\frac{8}{3} a^{2}\left|z_{2}\right|^{6}+p\left(\left|z_{1}\right|,\left|z_{2}\right|\right),
\end{aligned}
$$

where $a$ is a nonzero constant and $p$ is a homogeneous polynomial of degree 8 to be determined later. We define

$$
\omega=\frac{\sqrt{-1}}{2} \partial \bar{\partial} f=\frac{\sqrt{-1}}{2} \sum_{i, j} g_{i \bar{j}} d z_{i} \wedge d \bar{z}_{j} .
$$

It is straightforward to check that $\omega$ defines a Kähler metric $g$ if the ball is sufficiently small (note that the metric is not complete).

Direct computation gives

$$
\begin{aligned}
& g_{1 \overline{1}}=1+4 a\left|z_{1}\right|^{2}+8 a\left|z_{2}\right|^{2}+24 a^{2}\left|z_{1}\right|^{4} \\
& +112 a^{2}\left|z_{1}\right|^{2}\left|z_{2}\right|^{2}+28 a^{2}\left|z_{2}\right|^{4}+O\left(\left(\left|z_{1}\right|+\left|z_{2}\right|\right)^{6}\right), \\
& g_{12}=8 a \bar{z}_{1} z_{2}+56 a^{2} z_{1} \bar{z}_{1}^{2} z_{2}+56 a^{2} \bar{z}_{1} z_{2}^{2} \bar{z}_{2}+O\left(\left(\left|z_{1}\right|+\left|z_{2}\right|\right)^{6}\right), \\
& \text { and } g_{2 \overline{2}}=g_{1 \overline{1}} \text {. Therefore } \\
& \operatorname{det}\left(g_{i \bar{j}}\right)=g_{1 \overline{1}} g_{2 \overline{2}}-\left|g_{1 \overline{2}}\right|^{2} \\
& =\left(1+4 a\left|z_{1}\right|^{2}+8 a\left|z_{2}\right|^{2}+24 a^{2}\left|z_{1}\right|^{4}+112 a^{2}\left|z_{1}\right|^{2}\left|z_{2}\right|^{2}+28 a^{2}\left|z_{2}\right|^{4}\right)^{2} \\
& -\left|8 a \bar{z}_{1} z_{2}+56 a^{2} z_{1} \bar{z}_{1}^{2} z_{2}+56 a^{2} \bar{z}_{1} z_{2}^{2} \bar{z}_{2}\right|^{2}+O\left(\left(\left|z_{1}\right|+\left|z_{2}\right|\right)^{6}\right) \\
& =1+12 a\left(\left|z_{1}\right|^{2}+\left|z_{2}\right|^{2}\right)+84 a^{2}\left(\left|z_{1}\right|^{4}+\left|z_{2}\right|^{4}\right) \\
& +240 a^{2}\left|z_{1}\right|^{2}\left|z_{2}\right|^{2}+O\left(\left(\left|z_{1}\right|+\left|z_{2}\right|\right)^{6}\right) .
\end{aligned}
$$


Using $\log (1+x)=x-\frac{1}{2} x^{2}+O\left(x^{3}\right)$, we have

$$
\text { Ric }+12 a g=\partial \bar{\partial}\left(-\log \left(\operatorname{det} g_{i \bar{j}}\right)+12 a f\right)=\partial \bar{\partial} O\left(\left(\left|z_{1}\right|+\left|z_{2}\right|\right)^{6}\right) .
$$

Therefore Ric $+12 \mathrm{ag}$ vanishes up to order 3 at the origin. If we choose the function $p$ to be $-\lambda\left(\left|z_{1}\right|^{8}+\left|z_{2}\right|^{8}+8\left(\left|z_{1}\right|^{6}\left|z_{2}\right|^{2}+\left|z_{1}\right|^{2}\left|z_{2}\right|^{6}\right)\right)$, a direct computation gives

$$
\text { Ric }+12 a g=\partial \bar{\partial}\left(24 \lambda\left(\left|z_{1}\right|^{2}+\left|z_{2}\right|^{2}\right)^{3}+O\left(\left(\left|z_{1}\right|+\left|z_{2}\right|\right)^{6}\right)\right.
$$

where the term $O\left(\left(\left|z_{1}\right|+\left|z_{2}\right|\right)^{6}\right)$ does not depend on $\lambda$. If $\lambda$ is sufficiently large, Ric $+12 a g \geq 0$ near the origin. Set $K=-12 a$. Thus, near the origin, Ric $\geq K$. By direct computation $R_{1212}=R_{1313}=R_{1414}=4 a$ and $R_{1 u 1 v}=0$ at the origin if $u \neq v$. Combining this with the fact that the second derivatives of the Ricci tensor vanish at the origin we find, after a slight computation, that the fourth order term of (3-3) is greater than that of the complex space form if $e_{0}=\partial / \partial x_{1}$. So when $r$ is very small, $\sqrt{\operatorname{det}\left\langle J_{u}, J_{v}\right\rangle}$ is greater than that of the complex space form along the geodesic with initial direction $\partial / \partial x_{1}$ at the origin. Since

$$
\Delta r=\frac{\partial \log \sqrt{\operatorname{det}\left\langle J_{u}, J_{v}\right\rangle}}{\partial r},
$$

it follows that the pointwise Laplacian comparison with the complex space forms is not true for Kähler manifolds.

\section{Acknowledgments}

The author is grateful to his advisor Professor Jiaping Wang for continuous encouragement and helpful discussions during the work.

\section{References}

[Bishop and Crittenden 1964] R. L. Bishop and R. J. Crittenden, Geometry of manifolds, Pure and Applied Mathematics 15, Academic Press, New York, 1964. MR 29 \#6401 Zbl 0132.16003

[Cheeger and Ebin 2008] J. Cheeger and D. G. Ebin, Comparison theorems in Riemannian geometry, AMS Chelsea Publishing, Providence, RI, 2008. Revised reprint of the 1975 original. MR 2009c:53043 Zbl 1142.53003

[Gromov 1981] M. Gromov, Structures métriques pour les variétés riemanniennes, Textes Mathématiques 1, CEDIC, Paris, 1981. MR 85e:53051 Zbl 0509.53034

[Li 1993] P. Li, Lecture notes on geometric analysis, Lecture Notes Series 6, Research Institute of Mathematics, Seoul, 1993. MR 96m:58269 Zbl 0822.58001

[Li and Wang 2005] P. Li and J. Wang, "Comparison theorem for Kähler manifolds and positivity of spectrum”, J. Differential Geom. 69:1 (2005), 43-74. MR 2006g:53111 Zbl 1087.53067

[Schoen and Yau 1994] R. Schoen and S.-T. Yau, Lectures on differential geometry, Conference Proceedings and Lecture Notes in Geometry and Topology 1, International Press, Cambridge, MA, 1994. MR 97d:53001 Zbl 0830.53001 
Received March 14, 2011.

GANG LIU

DEPARTMENT OF MATHEMATICS

UNIVERSITY OF MINNESOTA

127 Vincent Hall, 206 ChURCH St. SE

MINNEAPOLIS, MN 55455

UNITED STATES

liuxx895@umn.edu 


\title{
PACIFIC JOURNAL OF MATHEMATICS
}

\author{
http://pacificmath.org \\ Founded in 1951 by \\ E. F. Beckenbach (1906-1982) and F. Wolf (1904-1989)
}

\section{EDITORS}

V. S. Varadarajan (Managing Editor)

Department of Mathematics

University of California

Los Angeles, CA 90095-1555

pacific@math.ucla.edu

Vyjayanthi Chari

Department of Mathematics

University of California

Riverside, CA 92521-0135

chari@math.ucr.edu

\section{Robert Finn}

Department of Mathematics Stanford University

Stanford, CA 94305-2125

finn@math.stanford.edu

Kefeng Liu

Department of Mathematics

University of California

Los Angeles, CA 90095-1555

liu@math.ucla.edu
Darren Long

Department of Mathematics

University of California

Santa Barbara, CA 93106-3080

long@math.ucsb.edu

Jiang-Hua Lu

Department of Mathematics

The University of Hong Kong

Pokfulam Rd., Hong Kong jhlu@maths.hku.hk

Alexander Merkurjev

Department of Mathematics

University of California

Los Angeles, CA 90095-1555

merkurev@math.ucla.edu
Sorin Popa

Department of Mathematics University of California

Los Angeles, CA 90095-1555 popa@math.ucla.edu

Jie Qing

Department of Mathematics

University of California

Santa Cruz, CA 95064

qing@cats.ucsc.edu

Jonathan Rogawski

Department of Mathematics

University of California

Los Angeles, CA 90095-1555

jonr@math.ucla.edu

\section{PRODUCTION}

pacific@math.berkeley.edu

\section{SUPPORTING INSTITUTIONS}

ACADEMIA SINICA, TAIPEI

CALIFORNIA INST. OF TECHNOLOGY INST. DE MATEMÁTICA PURA E APLICADA KEIO UNIVERSITY

MATH. SCIENCES RESEARCH INSTITUTE NEW MEXICO STATE UNIV.

OREGON STATE UNIV.

\author{
STANFORD UNIVERSITY \\ UNIV. OF BRITISH COLUMBIA \\ UNIV. OF CALIFORNIA, BERKELEY \\ UNIV. OF CALIFORNIA, DAVIS \\ UNIV. OF CALIFORNIA, LOS ANGELES \\ UNIV. OF CALIFORNIA, RIVERSIDE \\ UNIV. OF CALIFORNIA, SAN DIEGO \\ UNIV. OF CALIF., SANTA BARBARA
}

\author{
UNIV. OF CALIF., SANTA CRUZ \\ UNIV. OF MONTANA \\ UNIV. OF OREGON \\ UNIV. OF SOUTHERN CALIFORNIA \\ UNIV. OF UTAH \\ UNIV. OF WASHINGTON \\ WASHINGTON STATE UNIVERSITY
}

These supporting institutions contribute to the cost of publication of this Journal, but they are not owners or publishers and have no responsibility for its contents or policies.

See inside back cover or pacificmath.org for submission instructions.

The subscription price for 2011 is US \$420/year for the electronic version, and \$485/year for print and electronic.

Subscriptions, requests for back issues from the last three years and changes of subscribers address should be sent to Pacific Journal of Mathematics, P.O. Box 4163, Berkeley, CA 94704-0163, U.S.A. Prior back issues are obtainable from Periodicals Service Company, 11 Main Street, Germantown, NY 12526-5635. The Pacific Journal of Mathematics is indexed by Mathematical Reviews, Zentralblatt MATH, PASCAL CNRS Index, Referativnyi Zhurnal, Current Mathematical Publications and the Science Citation Index.

The Pacific Journal of Mathematics (ISSN 0030-8730) at the University of California, c/o Department of Mathematics, 969 Evans Hall, Berkeley, CA 94720-3840, is published monthly except July and August. Periodical rate postage paid at Berkeley, CA 94704, and additional mailing offices. POSTMASTER: send address changes to Pacific Journal of Mathematics, P.O. Box 4163, Berkeley, CA 94704-0163.

PJM peer review and production are managed by EditFLOW ${ }^{\mathrm{TM}}$ from Mathematical Sciences Publishers.

PUBLISHED BY PACIFIC JOURNAL OF MATHEMATICS

at the University of California, Berkeley 94720-3840

A NON-PROFIT CORPORATION

Typeset in LATEX

Copyright $(2011$ by Pacific Journal of Mathematics 


\section{PACIFIC JOURNAL OF MATHEMATICS}

Volume $254 \quad$ No. $2 \quad$ December 2011

Curvatures of spheres in Hilbert geometry

ALEXANDER BORISENKO and EUGENE OLIN

A formula equating open and closed Gromov-Witten invariants and its applications 275

to mirror symmetry

KWOKWAI CHAN

A note on $p$-harmonic $l$-forms on complete manifolds

LiAng-CHu CHANG and CHIUng-JUe ANNA SUNG

The Cheeger constant of curved strips

DAVID KREJČIŘíK and ALdo PRATELLI

Structure of solutions of 3D axisymmetric Navier-Stokes equations near maximal points

\section{ZHEN LEI and QI S. ZHANG}

Local comparison theorems for Kähler manifolds

GANG LIU

Structurable algebras of skew-rank 1 over the affine plane

SUSANNE PUMPLÜN

An analogue of Krein's theorem for semisimple Lie groups

SANJOY PUSTI

Une remarque de dynamique sur les variétés semi-abéliennes

GAËL RÉMOND

Fourier transforms of semisimple orbital integrals on the Lie algebra of $\mathrm{SL}_{2}$

LOREN SPICE

On noncompact $\tau$-quasi-Einstein metrics

LIN FENG WANG

Decomposition of de Rham complexes with smooth horizontal coefficients for semistable reductions

QIHONG XIE

A differentiable sphere theorem inspired by rigidity of minimal submanifolds 